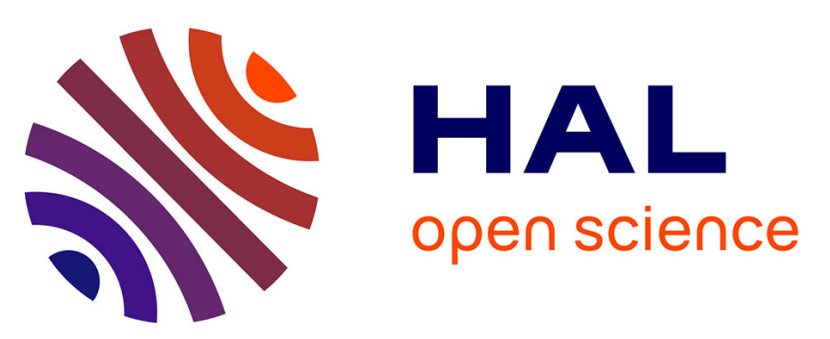

\title{
Modeling of Amiodarone Effect on Heart Rate Control in Critically Ill Patients with Atrial Tachyarrhythmias
}

Joe-Elie Salem, Maria El-Aissaoui, Margaux Alazard, Jean-Sébastien Hulot, Nadia Aissaoui, Jean-Yves Le-Heuzey, Christian Funck-Brentano, Christophe Faisy, Saik Urien

\section{To cite this version:}

Joe-Elie Salem, Maria El-Aissaoui, Margaux Alazard, Jean-Sébastien Hulot, Nadia Aissaoui, et al.. Modeling of Amiodarone Effect on Heart Rate Control in Critically Ill Patients with Atrial Tachyarrhythmias. Clinical Pharmacokinetics, 2016, pp.1-12. 10.1007/s40262-016-0372-9 . hal-01285452

\section{HAL Id: hal-01285452 https://hal.sorbonne-universite.fr/hal-01285452}

Submitted on 9 Mar 2016

HAL is a multi-disciplinary open access archive for the deposit and dissemination of scientific research documents, whether they are published or not. The documents may come from teaching and research institutions in France or abroad, or from public or private research centers.
L'archive ouverte pluridisciplinaire HAL, est destinée au dépôt et à la diffusion de documents scientifiques de niveau recherche, publiés ou non, émanant des établissements d'enseignement et de recherche français ou étrangers, des laboratoires publics ou privés. 


\section{Modeling of amiodarone effect on heart rate control in}

\section{critically ill patients with atrial tachyarrhythmias}

Running title: Amiodarone in critically ill patients with AT

Joe-Elie Salem, MD $\pitchfork \S$, Maria El-Aissaoui, MD $\ddagger$, Margaux Alazard, MD §\#, Jean-Sébastien Hulot, MD-PhD $\uparrow \S$, Nadia Aissaoui, MD-PhD $\ddagger$, Jean-Yves Le-Heuzey, MD-PhD **, Christian Funck-Brentano, MD-PhD $\uparrow \S$, Christophe Faisy* MD-PhD \|, Saik Urien*, MD-PhD $\|$.

* These authors contributed equally to this work

† AP-HP, Pitié-Salpêtrière Hospital, Department of Pharmacology and CIC-1421, F-75013 Paris, France

‡ Critical care Unit, Hôpital Européen Georges Pompidou, Assistance Publique-Hôpitaux de Paris, Université Paris Descartes Sorbonne Paris Cité, Paris, France.

$\S$ Sorbonne Universités, UPMC Univ Paris 06, Faculty of Medicine, INSERM UMRS ICAN1166, F-75013 Paris, France

I| Centre d'Investigation Clinique-1419 INSERM, EAU-08 Université Paris Descartes Sorbonne Paris Cité, Paris, France.

\# AP-HP, Pitié-Salpêtrière Hospital, Department of Cardiology - Rythmology unit, F-75013 Paris, France

** Cardiology - Rythmology Unit, Hôpital Européen Georges Pompidou, Assistance Publique-Hôpitaux de Paris, Université Paris Descartes Sorbonne Paris Cité, Paris, France.

Request for reprints and correspondence: Joe-Elie Salem, joe-elie.salem@aphp.fr, Centre d'Investigation Clinique Paris-Est, Hôpital La Pitié-Salpêtrière, Bâtiment Antonin Gosset, 47-83 Bld de I'hôpital, 75651 Paris Cedex 13, Secretariat : +33 1421785 31, Fax : 


\section{ABSTRACT}

Aims: Amiodarone is the gold standard medication to control heart rate in critically ill patients with atrial tachyarrhythmias (AT). However, effective doses and covariates influencing its efficacy remain unknown. We therefore performed pharmacodynamic modeling of heart rate reduction induced by amiodarone in these patients.

Methods-Results: This observational study included 80 consecutive severely ill patients receiving amiodarone to treat atrial tachyarrhythmias. A total of 1348 timeheart rate observations with 361 amiodarone dose administrations were analyzed during up to 6 days after hospital treatment initiation using a nonlinear mixed effect model. Pretreatment with amiodarone before intensive care administration, paroxysmal versus persistent AT, catecholamine infusion, fluid and magnesium loading were among covariates assessed in the model.

In case of paroxysmal AT in a patient not pretreated by amiodarone, a $300 \mathrm{mg}$ intravenous loading dose combined with a $800 \mathrm{mg}$ oral dose on the first day followed by $800 \mathrm{mg} /$ day p.o. for 4 days was effective to achieve a heart rate between 80 and $115 \mathrm{bpm}$ within the first day and to maintain it during the next 4 days. Corresponding doses were twice as high in patients with persistent AT. Use of intravenous magnesium $(p<0.02)$ and fluid loading $(p<0.02)$ was associated with an earlier and greater heart rate decrease. Dobutamine use had an opposite influence $(p<0.05)$.

Conclusions: In critically ill patients with AT, dose of amiodarone required to control heart rate is influenced by the type of AT and by other easily measurable conditions which may allow better individualization of amiodarone dosing.

Key words: amiodarone, atrial tachyarrhythmia, pharmacology, shock, heart rate.

\section{KEY POINTS}


1/ Amiodarone pre-treatment, paroxysmal versus persistent atrial tachyarrhythmias (AT), dobutamine infusion, intravenous magnesium and fluid loading have significant influence on amiodarone effect on heart rate reduction in critically ill patients with AT. 2/ An optimized dosing regimen of amiodarone refining current guidelines indications is proposed to better control heart rate in patients with AT depending on their clinical presentation: paroxysmal AT amiodarone naive, persistent AT amiodarone naive and paroxysmal AT amiodarone pre-treated.

3/ Co-administration of intravenous magnesium with amiodarone is associated with a greater and earlier heart rate control in patients with AT. This suggests that systematic use of IV magnesium as an add-on therapy might be valuable

\section{ABBREVIATION}

AT: atrial tachyarrhythmias

bpm: beats per minute

ECG: electrocardiogram

ECV : Electrical cardioversion

HR: heart rate

ICU: intensive care unit

IV : intravenous

mg: milligram 


\section{INTRODUCTION}

Atrial tachyarrhythmias (AT), such as atrial fibrillation are highly prevalent and are associated with a poor outcome in critically ill patients admitted in intensive care units $(\mathrm{ICU})^{[1]}$. AT is often characterized by a persistent elevation of ventricular heart rate (HR) above 120-130 beats per minute (bpm) leading to cardiac systolic dysfunction, (i.e ventricular tachycardiomyopathy), responsible for hemodynamic instability, increased morbidity and mortality ${ }^{[2-5]}$. A rate or rhythm control strategy with pharmacological therapy is recommended in patients with AT for the treatment or prevention of ventricular tachycardiomyopathy ${ }^{[4-5]}$. Before initiating antiarrhythmic treatment, factors promoting AT in the critically ill should be sought and corrected (anemia, hypoxia, hypokalemia...). Anticoagulant treatments are also usually administered. Attempts to restore sinus rhythm are frequently unsuccessful in the setting of critically ill patients ${ }^{[6-8]}$, particularly when using electrical cardioversion without antiarrhythmic pretreatment. HR control is thus the main preferred strategy in this situation ${ }^{[9-11]}$. Guidelines in stable patients recommend strict control aiming at a resting HR between $60-80 \mathrm{bpm}$ and $90-115 \mathrm{bpm}$ during stress situations, based on the results of the AFFIRM trial ${ }^{[4,12]}$. There are no specific guidelines for patients in severely ill condition but it is commonly admitted that reaching a HR below $115 \mathrm{bpm}$ is advisable ${ }^{[2-5]}$.

Amiodarone is the recommended agent to control HR in critically ill patients presenting with AT ${ }^{[4-5]}$. However, it can be associated with hypotension and potentially severe liver and pulmonary toxicity, particularly after IV administration ${ }^{[4,13-}$ 15]. This toxic risk justifies the administration of the lowest possible dose to control heart rate. In addition, patients admitted in ICU present with a diversity of adverse conditions that can affect antiarrhythmic drug pharmacokinetics and dynamics, 
including instable hemodynamics, pre-existent or acquired left ventricular dysfunction, hypoxemia, hyperthermia, hypovolemia or acidosis ${ }^{[16]}$.

The choice of amiodarone doses in ICU patients is thus challenging due to interpatient heterogeneity and limited knowledge of optimal dosing in this setting ${ }^{[4-5]}$. In general, an IV loading dose of $300 \mathrm{mg}$ is administered over 1 hour followed by an additional dose given either intravenously (IV) over the first day $(10-50 \mathrm{mg} / \mathrm{h})^{[10]}$ or orally over the next few days $(400 \mathrm{mg} / \text { day up to } 1200 \mathrm{mg} / \text { day })^{[4,5,17,18]}$. In daily practice, amiodarone dosing regimens used in these patients vary widely. Factors that influence amiodarone efficacy in critically ill patients have not been reported. Therefore, a randomized study comparing the efficacy of different amiodarone dosing regimens would be inappropriate without previous knowledge of the sources of variability of drug response in ICU patients. Modelling of amiodarone efficacy in critically ill patients has not been previously reported and is the best initial approach to better characterize the complex interactions of multiple covariates on amiodarone pharmacodynamics.

The objective of this study was to determine optimal doses of amiodarone to achieve a heart rate between 80 and $115 \mathrm{bpm}$ when treating AT after identification and integration of clinical and therapeutic covariates influencing amiodarone efficacy in critically ill patients. 


\section{MATERIAL AND METHODS}

\section{Patients and Study Design}

This observational, cohort study was conducted from January 2007 to April 2012 in the 18-bed medical ICU of a tertiary teaching hospital. Due to the observational design, and in accordance with French bioethics laws, Institutional Review Board authorization was not necessary. The study was approved by the Commission Nationale de I'Informatique et des Libertés to use computerized medical data with protection of patient confidentiality. Data were extracted from the files of 80 consecutive critically ill patients who had received at least one dose of amiodarone to treat or prevent AT during their hospitalization in ICU. Three investigators (JES, MEA, MA) were each responsible of the data extraction during a specified time period resulting in patients inclusion over three distinct time periods. [Figure1]. Patients with paced rhythm or incomplete chart were excluded. HR data were collected at the moment of the first amiodarone administration in ICU and then 4 to 6 times daily after each dosing over 6 days or until death or ICU discharge. Amiodarone could be administered either IV over 20 to 60 minutes or orally. Route, frequency of administration and doses were prescribed at the discretion of the treating physician without any predefined protocol.

\section{Variables}

At the time of first amiodarone administration in ICU the following time-independent covariates were collected: gender, age, body weight, height, etiology of the disease leading to ICU admission, Simplified Acute Physiology Score II (SAPS II score), Acute Physiology And Chronic Health Evaluation II (APACHE II score), diabetes, hypertension, past medical history of AT, withdrawal of a chronic antiarrhythmic drug since less than three times its half-life, total bilirubinemia, previous amiodarone treatment before first dose in ICU (yes, no), type of cardiac rhythm (sinus versus 
persistent / paroxysmal AT). Paroxysmal and persistent AT were defined as an arrhythmia which started less or more than one week before the first amiodarone administration in ICU, respectively.

Time-dependent covariates potentially interacting with amiodarone pharmacokinetics and/or pharmacodynamics were also collected at the time of each HR collection. These were: temperature, $\mathrm{pH}, \mathrm{pa02}$, natremia, kalemia, hemoglobin, protidemia, glomerular filtration rate, attempt to electrical cardioversion, magnesium or fluid loading, use of other antiarrhythmic drugs, furosemide or extra-renal dialysis with or without ultrafiltration, catecholamine administration and its type (epinephrine $<1 \mathrm{mg} / \mathrm{h}$, epinephrine $>1 \mathrm{mg} / \mathrm{h}$, dobutamine, other $\beta$-adrenoceptor agonists), curare and sedative drug use. Fluid loading was defined as administration of more than 0.5 liter of saline solution in 30 minutes. Magnesium loading was defined by any administration of IV magnesium either by bolus or continuous infusion whichever the dose received. Influence of other antiarrhythmic drugs could not be analyzed because such prescription was too rare to be studied.

\section{Pharmacodynamic Modeling}

General model

Amiodarone pharmacokinetics was ascribed to a virtual compartment model including zero or first order input rates. This virtual compartment, $A(t)$, represents the biophase in which the amount or concentration is in equilibrium with the observed effect. It is used to extract the kinetic component, KDE or "pharmacodynamic halflife", from the pharmacodynamic data alone describing the equilibrium between the administration rate and the observed effect. $\mathrm{G}(\mathrm{t})$ represents the amiodarone amount in the gut at a given time and ka, the first-order absorption rate (fixed to 8) [19-21]. 
Amiodarone bioavailability (F) was fixed to 0.33 [19-21]. The effect of amiodarone on heart rate, $\mathrm{HR}(\mathrm{t})$ was modeled as follows:

$$
\begin{gathered}
d A(t) / d t=\text { input }-K D E \times A(t) \\
\text { input }=k a \times G(t) \times F \text { if administered orally } \\
I R=K D E \times A(t) \text { and } E m a x=1 \\
d H R(t) / d t=k i n[1-E m a x \times I R /(I R+I R 50)]-\text { kout } H R(t)
\end{gathered}
$$

In the effect model, equation (4), the production rate constant of response HR, kin, is inhibited by the amiodarone virtual infusion rate, IR (drug amount per time unit) via an hyperbolic function. Finally, IR50 and kout are the IR that induces $50 \%$ of the maximal response and the first-order dissipation rate constant for the HR response. It was shown that the use of the IR function usually performs well and can be used for predictions of drug effects ${ }^{[26]}$, provided the investigated doses extend well beyond the IR50, which is the case in this study (Table 1).

The initial conditions of these differential equations were $\mathrm{HR}(0)=$ heart rate at time zero, $\mathrm{A}(0)=$ Dose for IV administration and $\mathrm{G}(0)=\mathrm{F} \times$ Dose for oral route. Influence of covariates

Several time-dependent variables were tested, and then included in the response model when they significantly (percent relative standard error $(\%$ rse) $<50 \%$ ) modified either kin (ModKin) or kout (ModKout) as follows :

$$
\mathrm{dHR}(\mathrm{t}) / \mathrm{dt}=\mathrm{M}_{\text {od }} \mathrm{K}_{\text {in }} \times k_{\text {in }}\left[1-\mathrm{IR}_{\text {Amio }} /\left(\mathrm{IR}_{\text {Amio }}+\operatorname{IR} \mathrm{R}_{\text {Amio }} 50\right)\right]-\mathrm{M}_{\text {od }} \mathrm{K}_{\text {out }} \times k_{\text {out }} \mathrm{HR}(\mathrm{t})
$$

with

$$
\begin{aligned}
& M_{\text {od }} K_{\text {in }}=\left(1-t_{M G} M G\right) \\
& M_{\text {od }} K_{\text {out }}=\left(1-t_{\text {DOBU DOBU }}\left(1+t_{\text {FILL }} \text { FILL }\right)\left(1+t_{E C V} \text { ECV }\right)(\text { RetroHR })\right.
\end{aligned}
$$

where MG, DOBU, FILL and ECV denote magnesium loading, beta-agonist administration, fluid therapy and electrical cardio-version (only when effectively 
restoring sinus rhythm), respectively, with their corresponding tsUBSCRIPT influential parameters.

where Retro ${ }_{\mathrm{HR}}=\left(\mathrm{HR} / \mathrm{HR}_{\mathrm{REF}}\right)^{\text {FRetroHR }}$

FRetro $_{\mathrm{HR}}$ denotes the parameter modulating the importance of spontaneous retrocontrol to a reference value, $\mathrm{HR}_{\mathrm{REF}}$, depending on the type of cardiac rhythm at the time of first amiodarone administration in ICU.

Several time-independent factors were tested and then included in the response model when they significantly (\%rse<50\%) modified either baseline HR before first amiodarone dose in ICU $\left(\mathrm{HR}_{(0)}\right)$ or $k_{\text {out }}$ or $\mathrm{HR}_{\mathrm{REF}}$ as follows:

$$
\begin{aligned}
& \mathrm{HR}_{(0)}=154 \times(\mathrm{Age} / 70)^{-0.23} \times\left[0.85^{\text {(if persistent AT) }} \text { or } 0.67^{\text {(if sinus rhythm) }}\right] \times 0.86^{\text {(if Amio pretreatment) }} \\
& k_{\text {out }}=0.9 \times 0.37^{\text {(if persistent AT or sinus rhythm) }} \\
& \mathrm{HR}_{\mathrm{REF}}=78.6 \times 1.73^{\text {(if persistent AT) }}
\end{aligned}
$$

Covariates effects on structural parameters were only tested and included if they were physiologically plausible. For example $\mathrm{HR}_{(0)}$ is known to be faster in patients with AT compared to patients in sinus rhythm, so this covariate effect can be accepted if it is significant.

The inclusion of patients in sinus rhythm was useful for they received the lowest amiodarone doses and thus served to validate the model over a large dose range. These subjects specificities were very well identified during the modeling process, since the "Sinus rhythm" covariate had a significant effect on the three major elements of our model : $\mathrm{HR}_{(0)}$ (i,e baseline heart rate at the time of amiodarone administration), Kout and ReferenceHR.

\section{Data analysis details}

Data was analyzed using the nonlinear mixed effect modeling software program Monolix version 4.1.3 (www.lixoft.com). Parameters were estimated by computing the maximum likelihood estimator of the parameters without any approximation of the 
model using the stochastic approximation expectation maximization algorithm combined to a Markov Chain Monte Carlo procedure. A combined, proportional plus constant, error model was used to describe residual variability and between-subject variability (BSV) was ascribed to an exponential error model. The Bayesian information criterion (BIC) was used to test different hypotheses regarding the final model, i.e., the covariate effects on parameters, residual variability model (proportional versus proportional plus additive error model), and structure of the variance-covariance matrix for the BSV parameters. Residuals were presented as normalized prediction distribution errors, based on the estimates of unbiased means and variances of the predictions by using 500 Monte Carlo simulations of the final model (the calculation includes a de-correlation step of the prediction errors). The mean of these normalized residues must not be different from 0 . Goodness-of-fit plots for the final model of amiodarone pharmacodynamics are represented in Figure 2. Results are expressed as numbers (\%), means \pm standard deviation, or medians (interquartile ranges) as appropriate. A p value $<0.05$ was considered significant. Simulation graphics and statistics were derived using the $\mathrm{R}$ software program. 


\section{RESULTS}

\section{Patients and Observations}

Among the 856 consecutive screened patients admitted to ICU, 97 (11.3\%) had received at least one amiodarone dose for AT. Seventeen patients had paced rhythm or incomplete chart and were therefore excluded, leaving 80 patients eligible for analysis [Figure 1]. In these 80 patients, 1348 time-heart rate observations with 361 amiodarone dose administrations were available during a maximum of 6 days after the first dosing in ICU. In our dataset during the observed period, patients with paroxysmal AT $(n=47)$ had heart rate above 115 bpm less frequently than patients with persistent AT ( $n=25)$ : respectively $37 \%$ (261/706) versus $47.7 \%(224 / 470)$ of HR collected $(p=0.02)$. Among patients initially diagnosed with AT in our dataset $(n=72)$, $33(46 \%)$ never returned to sinus rhythm while 39 had at least one ECG in sinus rhythm. Among those latter 39 patients, AT relapsed in 12 patients. Overall, sinus rhythm was restored in only 14 patients (19\%) within 24 hours of first amiodarone administration in ICU without recurrence of AT. Electrical cardioversion was delivered to 9 patients and successful in only 4 , of whom 2 after several attempts at distinct times of care. Those data confirm the difficulty to achieve and maintain heart rate control or sinus rhythm in clinical practice. More details about heart rate data collection and amiodarone doses are summarized in Table 1. Patients were severely ill, had cardiovascular comorbidities and received amiodarone during their ICU stay mainly for poorly tolerated paroxysmal atrial fibrillation. Dobutamine $(10 \%)$ or epinephrine (56\%) were the only IV catecholamine used. Before first amiodarone administration in ICU, 52 patients were naive of amiodarone pre-treatment $(74 \%$ patients with paroxysmal AT and $26 \%$ with persistent AT) and 28 patients had previously received amiodarone. Thirty patients (37.5\%) received magnesium loading 
as add-on therapy on top of amiodarone to control heart rate and only two patients received digoxin. The main clinical, demographic and biological patients' characteristics are summarized in Table2. Patients were mainly admitted for cardiogenic shock or sepsis. No stroke was identified during patients stay in ICU. Other treatments received by the patients that could interfere with heart rate control are summarized in Table2.

Impact of amiodarone pretreatment and type of atrial tachyarrhythmias on amiodarone doses needed to control heart rate.

Baseline heart rate before the first administration of amiodarone in ICU (defined as $\left.\mathrm{HR}_{(0)}\right)$ was higher (Table3) in amiodarone-naive patients with paroxysmal AT than in amiodarone-pretreated patients with paroxysmal AT or amiodarone-naive patients with persistent AT (154 \pm 4 vs. $132 \pm 35$ and $131 \pm 6$ bpm, respectively, $p<0.0001)$.

We modeled the effect of different amiodarone dosing regimen on heart rate reductions in these three different clinical scenarios and took the optimal dosing regimen to achieve a $\mathrm{HR}$ of $90 \mathrm{bpm}$ as an example (Figure3). The amiodarone dosages were then extrapolated from these simulations results.

Among patients with paroxysmal AT, slightly higher doses of amiodarone were needed to achieve a heart rate below $115 \mathrm{bpm}$ within the first day and to maintain it around $90 \mathrm{bpm}$ for the 4 subsequent days in amiodarone-naive compared to pretreated patients. This was due to the difference in baseline HR (Figure3A-3B). In silico simulations indicated that the amiodarone dosing regimen to achieve such heart rate control in amiodarone-naive patients with paroxysmal AT should be $300 \mathrm{mg}$ IV loading dose on day 1 plus $800 \mathrm{mg}$ oral dose from day 1 to day 5 (Figure3A). Corresponding doses indicated by the model were slightly lower in patients with paroxysmal AT pretreated by amiodarone: $300 \mathrm{mg}$ IV loading dose on day 1 plus $400 \mathrm{mg}$ oral dose from day 1 to day 5 (Figure3B). 
Interestingly, despite lower baseline $\mathrm{HR}$ in amiodarone-naive patients with persistent AT, corresponding doses were almost twice those of amiodarone-naive patients with paroxysmal AT (600 mg IV loading dose on day 1 and $1400 \mathrm{mg}$ oral doses from day 1 to day 5, Figure $3 C$ ). This relative resistance of persistent AT was associated to a decrease in the amiodarone effect $\left(\mathrm{k}_{\text {out }}\right)$ in patients with persistent AT compared to paroxysmal AT (Table3).

Impact of dobutamine infusion, electrical cardioversion, magnesium and fluid loading on amiodarone-effect.

We then assessed the effect of covariates on amiodarone effect. Among the covariates analyzed, electrical cardioversion $(p<0.05)$, absence of dobutamine infusion $(p<0.05)$, intravenous magnesium $(p<0.02)$ and fluid loading $(p<0.02)$ had a significant positive impact on amiodarone pharmacodynamics in ICU patients with AT (Appendix1). Use of furosemide, extra-renal dialysis, epinephrine infusion, curare and sedative drugs had no significant impact. Underlying pathologies, hemodynamic conditions, kidney function and baseline clinico-biological values (Table2) had neither significant impact. Figures $3 D, E$ and $F$ show that the use of magnesium and/or fluid loading on the first day were associated with a greater and earlier HR decrease than suggested by amiodarone pharmacodynamic half-life ( $3.33 \pm 0.9$ days, Table3). In contrast, efficacy of amiodarone on HR control decreased if dobutamine was used (Figures 3D, E and F). 


\section{DISCUSSION}

The present study indicates that amiodarone doses needed to control HR should be almost twice as high in persistent AT compared to paroxysmal $A T^{[4,19,22]}$. Patients who had received amiodarone before first ICU administration required lower doses. Our pharmacodynamic model also showed that the main interventional covariates influencing amiodarone efficacy were dobutamine use, electrical cardioversion, magnesium and fluid loading.

\section{A mixed oral and IV loading dose over a few days}

Due to incomplete bioavailability and delayed oral absorption ${ }^{[19-21]}$, a $300 \mathrm{mg} \mathrm{IV}$ loading dose is recommended ${ }^{[4,5]}$ to rapidly achieve the aimed HR. Thus, the total daily dose that may be given safely is expected to be higher with the oral route, avoiding hypotension, hemodynamic impairment and phlebitis which can specifically occur with IV amiodarone infusion ${ }^{[4,23-26]}$. Oral loading dosing regimen up to 30 to 50 $\mathrm{mg} / \mathrm{kg}$ over 5 days ${ }^{[27]}$ were proved safe and effective. In our model, we therefore chose to test a rapid and safe therapeutic strategy combining IV and oral dosing regimen over 5 days. According to our model, the different proposed dose scheme (300 to $600 \mathrm{mg}$ IV loading dose on day 1 plus 400 to $1400 \mathrm{mg}$ oral dose from day 1 to day 5) in patients with paroxysmal or persistent AT, amiodarone-pretreated or not, agree with the recommended doses ${ }^{[4,5,18,27]}$ but provide a more specific approach in each group.

Pharmacologic cardioversion with amiodarone is not effective in the short and medium term. At 24 hours, amiodarone has inconsistently demonstrated increased restoration of sinus rhythm when compared to placebo in some but not all randomized studies ${ }^{[4,5]}$. We therefore, focused on heart rate reduction which is a clinically relevant therapeutic endpoint in critically ill patients ${ }^{[4,5,10]}$. 


\section{Amiodarone pharmacodynamics in the ICU}

We identified four interventions that influenced amiodarone efficacy in critically ill patients with AT. Dobutamine use was associated with decreased amiodarone efficacy while electrical cardioversion, fluid and magnesium loading were associated with earlier and better HR control.

Fluid therapy, defined as infusion of more than 0.5 liter of saline administered over 30 minutes, was administered to patients with apparent or real hypovolemia and was significantly associated with better HR control. By correcting hypovolemia, reflex tachycardia may have decreased ${ }^{[28]}$. Dobutamine is a beta adrenergic agonist leading to tachycardia and supra-ventricular arrhythmias ${ }^{[29]}$ to a greater extent than other catecholamines, such as epinephrine ${ }^{[30]}$. In our model, dobutamine significantly influenced amiodarone pharmacodynamics while this interaction was not found with epinephrine. Interestingly, IV magnesium, which is recommended to prevent postoperative $A T^{[4,31]}$ was found to improve HR control of patients with AT treated with amiodarone. Further investigations are required to confirm the present findings before extrapolating our results to other patient populations and procedures for the treatment of AT. Electrical cardioversion (ECV) was rarely used or efficient to restore sinus rhythm in our patients. Ineffective or immediate recurrence of AT after ECV is an important concern in critically ill patients, and even more when AT is persistent ${ }^{[4]}$. ECV success rates as low as $30 \%$ are reported in these patients ${ }^{[32,33]}$. Impregnation with antiarrhythmic drugs such as amiodarone increases the probability to restore and maintain sinus rhythm ${ }^{[4,34,35]}$.

There was no significant influence of etiology of shock, sedative and curare drugs on amiodarone efficacy. Neither furosemide administration nor ultrafiltration were identified as significant covariates. We hypothesize that patients treated with these depletive strategies had high pre-therapeutic filling pressures and did not experience 
hypovolemia and reactional HR increase ${ }^{[28]}$. Type of atrial tachyarrhythmia was not a significant covariate but caution is needed because patients included in this modelling were mostly patients with atrial fibrillation and few data were available for other types of arrhythmia such as atrial flutter. Amiodarone and its metabolite $\mathrm{N}$-desethylamiodarone are eliminated by hepatic route and marginally by the kidney. In patients on hemodialysis or intermittent peritoneal dialysis, amiodarone and its metabolite were not found in the dialysate ${ }^{[36]}$. In the present study, we did not find any influence of creatinine clearance or extra-renal dialysis on the model parameters. Beta-blockers and verapamil were used exceptionally in this study because of their negative inotropic effects. Digoxin was also very rarely used because of its poor efficacy to rapidly slow $\mathrm{HR}$ and of significant interaction with amiodarone that may lead to adverse events ${ }^{[4,5]}$.

\section{Limitations}

In this observational study, we may have not taken into consideration other unavailable covariates of interest which may influence drug response. However, this study provides for the first time the basis for designing a future prospective trial of amiodarone dosing in ICU patients with AT. Also, the therapeutic strategy based on our model should now be prospectively tested to compare if a tailored amiodarone dosing strategy according to duration of AT (paroxysmal versus persistent) and other significant covariates (amiodarone pretreatment or not, coadministration of magnesium or not, on/off dobutamine) is more efficient and more convenient (less bradycardia and hypotension issues) to achieve an optimal heart rate control than a lenient strategy relying on physician's decisions without any standardized protocol.

Left atrial volume is inversely correlated to amiodarone dose needed to restore or maintain sinus rhythm after $A T^{[37]}$. To date, influence of left atrial volume on the 
ability of amiodarone to control heart rate is still unclear ${ }^{[18,22,38,39]}$. Impact of left atrial size could not be tested in our model due to incomplete data in too many patients.

Plasma levels of amiodarone and its metabolite N-desethylamiodarone, were not tested in our model because amiodarone therapeutic drug monitoring is not recommended ${ }^{[4,5]}$ in clinical practice. It is theoretically conceivable that including plasma concentrations of amiodarone and $\mathrm{N}$-desethylamiodarone could have improved our predictions. It is however unlikely since individual plasma concentrations are poorly predictive of pharmacodynamics response ${ }^{[40,41]}$

Our patients with paroxysmal AT pretreated with amiodarone had very heterogeneous doses before first administration in ICU. Due to our observational design, these pre-admission doses could not be precisely identified. In our model, we found that this covariate significantly influenced baseline HR. Baseline HR in this subgroup of patients were quite scattered (132 $\pm 35 \mathrm{bpm})$ compared to other subgroups and lower than in amiodarone naïve patients with paroxysmal AT $(154 \pm 3$ bpm). This difference in baseline HR between those latter groups could be explained by the fact that patients pretreated by amiodarone were already under its slowing effect on heart rate. Thus, our recommended doses of amiodarone for this subgroup of amiodarone-pretreated patients with paroxysmal AT was the lowest proposed and will be applicable only if baseline HR are in the 130-135 bpm range. Even lower doses would be needed if this baseline HR is lower than $120 \mathrm{bpm}$ and higher doses would be needed in patients with baseline HR above $140 \mathrm{bpm}$.

\section{Perspectives}

This work, modeling amiodarone efficacy to control HR in critically ill patients with AT, identified several clinically relevant covariates that influenced its pharmacodynamics. Taking into account these covariates might achieve better individualization of amiodarone dosing for poorly tolerated AT in critically ill patients. 
Compared to paroxysmal AT, double doses are needed in persistent AT. Amiodarone pretreated patients before ICU admission needed lower doses than amiodaronenaive patients. Correction of hypovolemia, magnesium administration and use of lower dobutamine doses also contributed to a better control of HR.

This study suggests that co-administration of magnesium with amiodarone is associated with a greater and earlier HR control in critically ill patients with AT. Lower amiodarone doses might be needed to slow AT in case of co-administration of magnesium, thereby potentially lowering the incidence of amiodarone adverse events. Administration of high doses of magnesium versus placebo as an adjunct to ibutilide to treat AT, a class III agent, was associated with a higher conversion rate to sinus rhythm and less ventricular arrhythmia ${ }^{[42]}$. To date, prophylactic use of IV magnesium has been shown to reduce the probability of post-operative $A T^{[31]}$ but data regarding its efficacy in acute constituted AT remain scarce, particularly when combined with amiodarone. We are planning to further investigate this issue by conducting a prospective study evaluating IV magnesium as add-on therapy to amiodarone to treat AT in critically ill patients. 


\section{CONCLUSION}

In critically ill patients with AT, our results indicate that doses of amiodarone needed to control heart rate are higher in persistent than in paroxysmal AT. In contrast, patients who had received amiodarone before first ICU administration required lower doses. In critically ill patients, optimization of fluid therapy, decreasing dobutamine infusion as soon as possible and magnesium supplementation may also help control heart rate in AT.

\section{FUNDING SOURCES}

No external funding was used in the preparation of this manuscript.

\section{DISCLOSURES}

JES, MEA, MA, JSH, NA, JYLH, CFB, CF, SU declare that they have no conflict of interest. 


\section{REFERENCES}

1. Artucio H, Pereira M. Cardiac arrhythmias in critically ill patients: epidemiologic study. Crit Care Med. 1990; 18:1383-8.

2. Packer DL, Bardy GH, Worley SJ et al. Tachycardia-induced cardiomyopathy: a reversible form of left ventricular dysfunction. Am J Cardiol.1986; 57:563-70.

3. Nerheim P, Birger-Botkin S, Piracha L, Olshansky B. Heart failure and sudden death in patients with tachycardia-induced cardiomyopathy and recurrent tachycardia. Circulation. $2004 ; 110: 247-52$

4. European Heart Rhythm Association, European Association for Cardio-Thoracic Surgery. Guidelines for the management of atrial fibrillation: the Task Force for the Management of Atrial Fibrillation of the European Society of Cardiology (ESC). Eur Heart J. 2010; 31:2369-429.

5. $2014 \mathrm{AHA} / \mathrm{ACC} / \mathrm{HRS}$ guideline for the management of patients with atrial fibrillation: a report of the American College of Cardiology/American Heart Association Task Force on Practice Guidelines and the Heart Rhythm Society. Circulation. 2014;130:2071-104. 2014; 64:e1-76.

6. Kumar A. Intravenous amiodarone for therapy of atrial fibrillation and flutter in critically ill patients with severely depressed left ventricular function. South Med J. 1996; 89:779-85.

7. Clemo HF, Wood MA, Gilligan DM, Ellenbogen KA. Intravenous amiodarone for acute heart rate control in the critically ill patient with atrial tachyarrhythmias. Am J Cardiol. 1998; 81:594-8.

8. Mayr A, Knotzer H, Mutz N, Hasibeder W. Atrial tachyarrhythmia after cardiac surgery. Intensive Care Med. 1999; 25:242-3.

9. Galve E, Rius T, Ballester R, et al. Intravenous amiodarone in treatment of recentonset atrial fibrillation: results of a randomized, controlled study. J Am Coll Cardiol. 1996; 27:1079-82. 
10. Delle Karth G, Geppert A, Neunteufl T et al. Amiodarone versus diltiazem for rate control in critically ill patients with atrial tachyarrhythmias. Crit Care Med. 2001; 29:1149-53.

11. Carlsson J, Miketic S, Windeler J et al; STAF Investigators. Randomized trial of ratecontrol versus rhythm-control in persistent atrial fibrillation: the Strategies of Treatment of Atrial Fibrillation (STAF) study. J Am Coll Cardiol. 2003; 41:1690-6.

12. Van Gelder IC, Hagens VE, Bosker HA et al; Rate Control versus Electrical Cardioversion for Persistent Atrial Fibrillation Study Group. A comparison of rate control and rhythm control in patients with recurrent persistent atrial fibrillation. $\mathrm{N}$ Engl J Med. 2002; $347: 1834-40$.

13. Donaldson L, Grant IS, Naysmith MR, Thomas JS. Acute amiodarone-induced lung toxicity. Intensive Care Med. 1998; 24:626-30.

14. Rätz-Bravo AE, Drewe J, Schlienger RG, Krähenbühl S, Pargger H, Ummenhofer W. Hepatotoxicity during rapid intravenous loading with amiodarone: Description of three cases and review of the literature. Crit Care Med. 2005; 33:128-34; discussion 245-6.

15. Papiris SA, Triantafillidou C, Kolilekas L, Markoulaki D, Manali ED. Amiodarone: review of pulmonary effects and toxicity. Drug Saf. 2010; 33:539-58.

16. Lafont E, Urien S, Salem J.E, Heming N, Faisy C. Modeling for critically ill patients: An introduction for beginners. Journal of Critical Care 2015 (« In press »).

17. Tonet JL, Lechat $\mathrm{P}$, Frank $\mathrm{R}$ et al. Electrocardiographic effects and antiarrhythmic action of $1200 \mathrm{mg}$ of oral amiodarone per day. Ann Cardiol Angeiol (Paris). 1984; 33:309-15. 18. Kochiadakis GE, Igoumenidis NE, Solomou MC, Kaleboubas MD, Chlouverakis GI, Vardas PE. Efficacy of amiodarone for the termination of persistent atrial fibrillation. Am J Cardiol. 1999; 83:58-61.

19. Anastasiou-Nana M, Levis GM, Moulopoulos S. Pharmacokinetics of amiodarone after intravenous and oral administration. Int J Clin Pharmacol Ther Toxicol. 1982; 20:524-9. 20. Pourbaix S, Berger Y, Desager JP, Pacco M, Harvengt C. Absolute bioavailability of amiodarone in normal subjects. Clin Pharmacol Ther. 1985; 37:118-23. 
21. Daily Med, Current Medication Information, Amiodarone. http://dailymed.nlm.nih.gov/dailymed/index.cfm

22. Vardas PE, Kochiadakis GE, Igoumenidis NE et al. Amiodarone as a first-choice drug for restoring sinus rhythm in patients with atrial fibrillation: a randomized, controlled study. Chest. 2000; 117:1538-45.

23. Lotto A, Satolli R, Baldini MR. Hemodynamic effects of amiodarone. Circulation. $1980 ; 62: 666$.

24. Schwartz A, Shen E, Morady F et al. Hemodynamic effects of intravenous amiodarone in patients with depressed left ventricular function and recurrent ventricular tachycardia. Am Heart J. 1983; 106:848-56.

25. Remme WJ, Kruyssen HA, Look MP et al. Hemodynamic effects and tolerability of intravenous amiodarone in patients with impaired left ventricular function. Am Heart J. 1991; 122:96-103.

26. Jacqmin P, Snoeck E, van Schaick EA et al. Modelling response time profiles in the absence of drug concentrations: definition and performance evaluation of the KPD model. J Pharmacokinet Pharmacodyn. 2007;34:57-85.

27. Evans SJ, Myers M, Zaher $\mathrm{C}$ et al. High dose oral amiodarone loading: electrophysiologic effects and clinical tolerance. J Am Coll Cardiol. 1992; 19:169-73.

28. Hainsworth R. Reflexes from the heart. Physiol Rev. 1991; 71:617-58.

29. Fellahi J-L, Parienti J-J, Hanouz J-L et al. Perioperative use of dobutamine in cardiac surgery and adverse cardiac outcome: propensity-adjusted analyses. Anesthesiology. 2008; 108:979-87.

30. Butterworth J. Dobutamine: too dangerous for « routine » administration? Anesthesiology. 2008; 108:973-4.

31. Miller S, Crystal E, Garfinkle M et al. Effects of magnesium on atrial fibrillation after cardiac surgery: a meta-analysis. Heart. 2005; 91:618-23. 
32. Mayr A, Ritsch N, Knotzer $\mathrm{H}$ et al. Effectiveness of direct-current cardioversion for treatment of supraventricular tachyarrhythmias, in particular atrial fibrillation, in surgical intensive care patients. Crit Care Med. 2003; 31:401-5.

33. Kanji S, Williamson DR, Yaghchi BM et al. Epidemiology and management of atrial fibrillation in medical and noncardiac surgical adult intensive care unit patients. J Crit Care. 2012; 27:326.e1-8.

34. Singh BN, Singh SN, Reda DJ et al; Sotalol Amiodarone Atrial Fibrillation Efficacy Trial (SAFE-T) Investigators. Amiodarone versus sotalol for atrial fibrillation. N Engl J Med. $2005 ; 352: 1861-72$.

35. Sticherling C, Behrens S, Kamke W, Stahn A, Zabel M. Comparison of acute and long-term effects of single-dose amiodarone and verapamil for the treatment of immediate recurrences of atrial fibrillation after transthoracic cardioversion. Europace. 2005; 7:546-53.

36. Harris L, Hind CR, McKenna WJ et al. Renal elimination of amiodarone and its desethyl metabolite. Postgrad Med J. 1983; 59:440-2.

37. Vietti-Ramus G, Veglio F, Marchisio U, Burzio P, Latini R. Efficacy and safety of short intravenous amiodarone in supraventricular tachyarrhythmias. Int J Cardiol. 1992; 35:77-85.

38. Brodsky MA, Allen BJ, Walker CJ et al. Amiodarone for maintenance of sinus rhythm after conversion of atrial fibrillation in the setting of a dilated left atrium. Am J Cardiol. 1987; $60: 572-5$

39. Kochiadakis GE, Igoumenidis NE, Parthenakis FI, Chlouverakis GI, Vardas PE. Amiodarone versus propafenone for conversion of chronic atrial fibrillation: results of a randomized, controlled study. J Am Coll Cardiol. 1999; 33:966-71

40. Greenberg ML, Lerman BB, Shipe JR, Kaiser DL, DiMarco JP. Relation between amiodarone and desethylamiodarone plasma concentrations and electrophysiologic effects, efficacy and toxicity. J Am Coll Cardiol. 1987; 9:1148-55.

41. Deharo JC, Durand A, Macaluso G et al. Clinical electrophysiologic effects of a single high oral dose of amiodarone. Fundam Clin Pharmacol. 1997; 11:275-80. 
42. Patsilinakos $\mathrm{S}$, Christou $\mathrm{A}$, Kafkas $\mathrm{N}$ et al. Effect of high doses of magnesium on converting ibutilide to a safe and more effective agent. Am J Cardiol. 2010; 106:673-6. 


\section{FIGURES AND LEGENDS}

Figure 1. Flow chart of all the screened patients

Figure 2. Goodness-of-fit plots for the final model of amiodarone pharmacodynamics.

The following data illustrate the robustness of the pharmacodynamic model. You can see the observed versus model-predicted heart rate for individual predictions $(A)$ and normalized prediction distribution errors (NPDE) versus predicted heart rates over time (B). The solid lines represent the identity lines and the dashed blue line represents the regression lines. Correlation between observed and individual predicted heart rate is $r: 0.84$. The mean of the NPDE residuals was not significantly different from 0 ( $p$ : 0.32). Prediction-corrected visual predictive check for heart rate over time in days are presented in figure $\mathrm{C}$. The green lines are the median and quartiles $\left(25^{\text {th }}\right.$ and $\left.75^{\text {th }}\right)$ of observed values, with the areas surrounding them representing the $90 \%$ confidence intervals of the corresponding simulated quartiles. 


\section{Figure 3. Model-predicted effect of amiodarone (Amio) on heart rate (HR) in beats per minutes (bpm).}

Shown are the effects in case of paroxysmal atrial tachyarrhythmias (AT) in amiodarone (Amio) naive patients before first administration in ICU (A,D, baseline HR $154 \mathrm{bpm}$ ), paroxysmal AT in patients pretreated by Amio (B,E, baseline HR $132 \mathrm{bpm}$ ), and persistent AT in patients naive of Amio(C,F, baseline HR $131 \mathrm{bpm}$ ). In figure A, B and C, are represented the evolution of HR over five days following several doses of Amio. Shown are the effects for a $300 \mathrm{mg}$ intravenous(IV) loading dose on day 1 (D1) combined with a 200/400/600/800/1200 mg (purple, green, light blue, dark blue and dark line respectively) oral dose on the first day followed by an equivalent oral doses for 4 days(D1-5). The orange line represent the effect for a $600 \mathrm{mg}$ IV loading dose on D1 combined with a $1400 \mathrm{mg}$ oral dose on the first day followed by an equivalent oral doses for 4 days(D1-5). Compared to the case of an amio naive patient with paroxysmal AT (A), doses needed to achieve HR between 80 and 115 bpm, are lower in patients with paroxysmal AT pretreated by Amio (B) and higher in amio naive patients with persistent $A T \operatorname{Amio}(C)$. In figure $A, B$ and $C$, the bold line represent the proposed dose scheme to use in clinical practice according to the situation (300/300/600 mg IV loading dose plus 800/400/1400 oral dose respectively in Figure A, B and C). This latter dose scheme (Figure A, B and $C$ ) is used in figure $D, E$ and $F$ respectively for further evaluation. In figure $D, E$ and $F$, are represented the evolution of HR over the first day following a selected Amio dose alone $(-)$ or in case of concomitant dobutamine (DOBU) use $(+++)$, fluid therapy (Fill) over 12 hours (……..) or/and magnesium (Mg) loading over 24 hours: for Mg alone (- - -), for combined Mg and Fill (- - ' - '). Use of magnesium or/and fluid loading the first day, as add-on therapy on Amio were associated with a faster and greater HR decrease. Efficacy of Amio on HR control decreased in case of persistent AT or if dobutamine was used. 


\section{TABLES}

Table 1. Heart rhythm characteristics and amiodarone dosing of the 80 patients

\section{Characteristic}

\section{Heart rate}

Total number of HR collected

Median time between consecutive HR collected, hours

Mean time between consecutive HR collected, hours

Median time between first and last HR collected, days

Mean time between first and last HR collected, days

Minimum/Maximum time between first and last HR collected, days

Median number of HR collected by patient

Mean number of HR collected by patient

\section{Amiodarone doses received}

Total number of amiodarone administrations

Patients with at least 1 IV dose/First dose delivered IV

Median amiodarone dose (IV route), $\mathrm{mg} /$ dose

Minimum/Maximum amiodarone dose (IV route), $\mathrm{mg} / \mathrm{dose}$

Minimum/Maximum amiodarone dose (IV route), $m g / d a y$

Patients with at least 1 oral dose / Nasogastric tube ( $\geq 1$ dose)

Median amiodarone dose (oral), $\mathrm{mg} /$ dose

Minimum/Maximum amiodarone dose (oral), $\mathrm{mg} /$ dose

Minimum/Maximum amiodarone dose (oral), $m g /$ day

Median time between each dose, days
1348 361

Value

$5.3(4.1-7.7)$

$6 \pm 2.8$

$5.3(3.5-5.9)$

$4.6 \pm 1.8$

$0.8 / 6.4$

19(12-22)

$16.9 \pm 7.3$

15(19\%)/11(14\%)

$300(175-300)$

$150 / 300$

$150 / 600$

$76(95 \%) / 51(64 \%)$

$400(200-1200)$

$100 / 2400$

$100 / 4200$

$0.94(0.49-1.01)$

Abbreviations: HR, Heart rate; IV, intravenous; mg, milligram

Statistics: Values are given as the mean \pm standard deviation, median (interquartile range 25-75) or number (\%). 
Table 2. Main Characteristics of the 80 patients studied

\section{Characteristic}

Value

\section{Demographic status and comorbidities}

Age, year

$75 \pm 13$

Male sex

$44(55 \%)$

Body weight, kilogram

$72 \pm 20$

Diabetes/Hypertension

$20(25 \%) / 41(51 \%)$

Coronary ischemic disease

$36(45 \%)$

Previous history of cardiac insufficiency

$31(39 \%)$

Previous history of supra-ventricular tachyarrhythmia

$22(28 \%)$

Chronic obstructive pulmonary disease

$23(29 \%)$

Chronic renal insufficiency (Clearance $<60 \mathrm{ml} / \mathrm{min} / \mathrm{m}^{2}$ )

$19(24 \%)$

APACHEII/SAPSII at admission in ICU

$27 \pm 9 / 59 \pm 20$

ICU mortality

$39(49 \%)$

Bio-clinical values at first administration of amiodarone in ICU

Mean arterial pressure $<65 \mathrm{mmHg}$

$29(36 \%)$

Body Temperature, $\left({ }^{\circ} \mathrm{Celsius}\right.$, eardrum)

$37.1(36.4-38 \%)$

Non-invasive/invasive mechanical ventilation

$9(11 \%) / 43(54 \%)$

Cardiac systolic dysfunction (Ejection fraction<45\%)

$31(39 \%)$

Sepsis

$35(44 \%)$

Paroxysmal/persistent atrial tachyarrhythmia

$47(59 \%) / 25(31 \%)$

Sinus rhythm

$8(10 \%)$

$\mathrm{pH}$

$7.4(7.26-7.43)$

$\mathrm{P}_{\mathrm{a}} \mathrm{O}_{2}(m m H g)$

$85(72-100)$

$\mathrm{Na}^{+}(\mathrm{mmol} / \mathrm{l})$

$137.5(135-141)$

$\mathrm{K}^{+}(\mathrm{mmol} / \mathrm{l})$

$3.7(3.2-4.6)$

Hemoglobin

10.9(9.1-12) 
Total bilirubin ( $\mathrm{mmol} / \mathrm{l})$

Prior antiarrhythmic treatment before first administration of amiodarone in ICU

Chronic antiarrhythmic treatment recently withdrawn

Chronic amiodarone intake (>1 month of continuous intake)

Sub-acute amiodarone pre-treatment ( $<4$ grams in the last month)

Amiodarone-naive (>3 months without amiodarone)

\section{Additional treatments after first amiodarone administration in ICU}

Attempted/Successful electrical cardioversion

Magnesium loading

Catecholamine ( $\beta$-agonists) : Dobutamine/Salbutamol

Catecholamine ( $\alpha$ and $\beta$-agonist) : Epinephrine

Catecholamine ( $\alpha$ agonist) : Norepinephrine

Fluid loading / duration longer than 12 hours $54(67.5 \%) / 11(14 \%)$

Renal replacement therapy/Diuretics $20(25 \%) / 40(50 \%)$

Sedative drugs/Curare $44(55 \%) / 20(25 \%)$

Other anti-arrhythmic (digoxin, beta blocker, verapamil)

Abbreviations: APACHEII, Acute Physiology And Chronic Health Evaluation at intensive care unit admission; ICU, intensive care unit ; SAPSII, simplified acute physiology score at intensive care unit admission

Statistics: Values are given as the mean \pm standard deviation, median (interquartile range 25-75) or number (\%).

* TSH was available in 35 subjects within 10 days before first administration of amiodarone in ICU. Among them, none had a TSH below lower normal limit $[<0.2 \mathrm{mUi} / \mathrm{L}]$ and three had a TSH above upper normal limit $[>5.2 \mathrm{mUi} / \mathrm{L}]$. 
Table 3. Parameter estimates of the final model of amiodarone effect on heart rate in the 80 critically ill patients studied

\begin{tabular}{|c|c|c|c|}
\hline Parameter & $\begin{array}{c}\text { Estimate } \\
(\% \text { rse })\end{array}$ & $\begin{array}{c}\text { Group Value, } \\
\text { units (\%rse) }\end{array}$ & $\begin{array}{l}\text { BSV } \\
\text { (\%rse) }\end{array}$ \\
\hline Pharmacodynamic Half-life $[\log (2) / K D E]$, day & $3.33(27)$ & 3.33 days $(27)$ & NA \\
\hline $\begin{array}{l}\text { HR }_{(0)} \text {, (if Parox AT, amiodarone naive), bpm } \\
\times \text { by Age effect, [(Age/70 })^{-0.23} \text { ] } \\
\times \text { if Persistent AT [vs parox AT] } \\
\times \text { if Sinus rhythm [vs parox AT] } \\
\times \times \text { if pretreated Amio [vs Amio naive] }\end{array}$ & $\begin{array}{l}154(3) \\
-0.23(41) \\
0.85(27) \\
0.67(18) \\
0.86(29)\end{array}$ & $\begin{array}{l}154 \text { bpm (3) } \\
131 \mathrm{bpm}(4) \\
103 \text { bpm (20) } \\
132 \text { bpm (26) }\end{array}$ & $0.14(11)$ \\
\hline $\begin{array}{l}\boldsymbol{k}_{\text {out }} \text { (if Parox AT), day }{ }^{-1} \\
\times \text { if Persistent AT or sinus rhythm [vs parox AT] }\end{array}$ & $\begin{array}{l}0.9(28) \\
0.37(48)\end{array}$ & $\begin{array}{l}0.9 \text { day }^{-1}(28) \\
0.33 \text { day }^{-1} \text { (NA) }\end{array}$ & $1.42(13)$ \\
\hline $\begin{array}{l}\text { Reference HR, (if Parox AT or sinus rhythm), bpm } \\
\quad \times \text { if Persistent AT [vs parox AT] }\end{array}$ & $\begin{array}{l}78.6(20) \\
1.73(47)\end{array}$ & $\begin{array}{l}78.6 \mathrm{bpm}(20) \\
136 \mathrm{bpm}(29)\end{array}$ & $0.8(11)$ \\
\hline FRetro $_{\mathrm{HR}}$ & $0.46(21)$ & & NA \\
\hline Amio $_{50}, \mathrm{mg} / \mathrm{day}$ & $291(29)$ & & NA \\
\hline $\begin{array}{l}t_{\mathrm{MG}} \\
t_{\mathrm{FILL}} \\
t_{\mathrm{ECV}} \\
t_{\text {DOBU }}\end{array}$ & $\begin{array}{c}0.4(32) \\
0.31(31) \\
0.37(31) \\
0.26(46)\end{array}$ & & \\
\hline Residual variability, bpm & $16(2)$ & & \\
\hline
\end{tabular}

Abbreviations: $\mathbf{A T}=$ Atrial fibrillation; $\mathbf{A m i o}_{50}=$ Amiodarone dosage that induces $50 \%$ of putative maximal effect on HR reduction; $\mathbf{D O B U}=$ beta-agonist administration; $\mathbf{b p m}=$ beats per minute; BSV = between-subject variability; ECV : electrical cardio-version; FILL = fluid therapy; FRetro $_{\mathrm{HR}}=$ factor determining the importance of endogenous spontaneous retrocontrol of the HR to a reference HR depending on the type of cardiac rhythm at the time of first administration of amiodarone in ICU; $\mathbf{H R}_{\mathbf{0}}=$ heart rate at $\mathrm{t}_{0}$ before first amiodarone dosing in ICU; ICU = intensive care unit; $\mathbf{K D E}=$ rate constant describing the equilibrium between the administration rate and the observed effect, $\boldsymbol{k}_{\text {out }}=$ first-order constant rate for Amio effect kinetics; \%rse $=$ percent relative standard error; $\mathbf{N A}=$ not applicable; $\mathbf{M G}=$ magnesium loading; Parox = Paroxystic atrial tachyarrhythmia which started less than one 
week before the first amiodarone administration in ICU; Reference HR = Estimated heart rate that the subject tend to reach spontaneously by endogenous retrocontrol; vs = versus. 


\section{FIGURE 1}

\section{Chart screening : 856 consecutive patients admitted in intensive care unit}

$18 / 01 / 2007$ to $20 / 07 / 2007$ (307 patients)

$12 / 01 / 2009$ to $09 / 11 / 2009$ ( 359 patients)

05/01/2012 to $07 / 04 / 2012$ (190 patients)

759 patients who never took amiodarone or took amiodarone for ventricular arrhythmia

\section{7 patients $(11,3 \%)$ administered amiodarone at least once for atrial tachyarrhythmia during hospitalization}

\section{Final Analysis : 80 patients administered} amiodarone for atrial tachyarrhythmia

- 8 for sinus rhythm maintenance

- 72 for pharmacological cardioversion or heart rate control

$91 \%$ atrial fibrillation, $5 \%$ flutter, $4 \%$ atrial tachyarrhythmia

Abbreviation : ECG , Electrocardiogram 
FIGURE 2
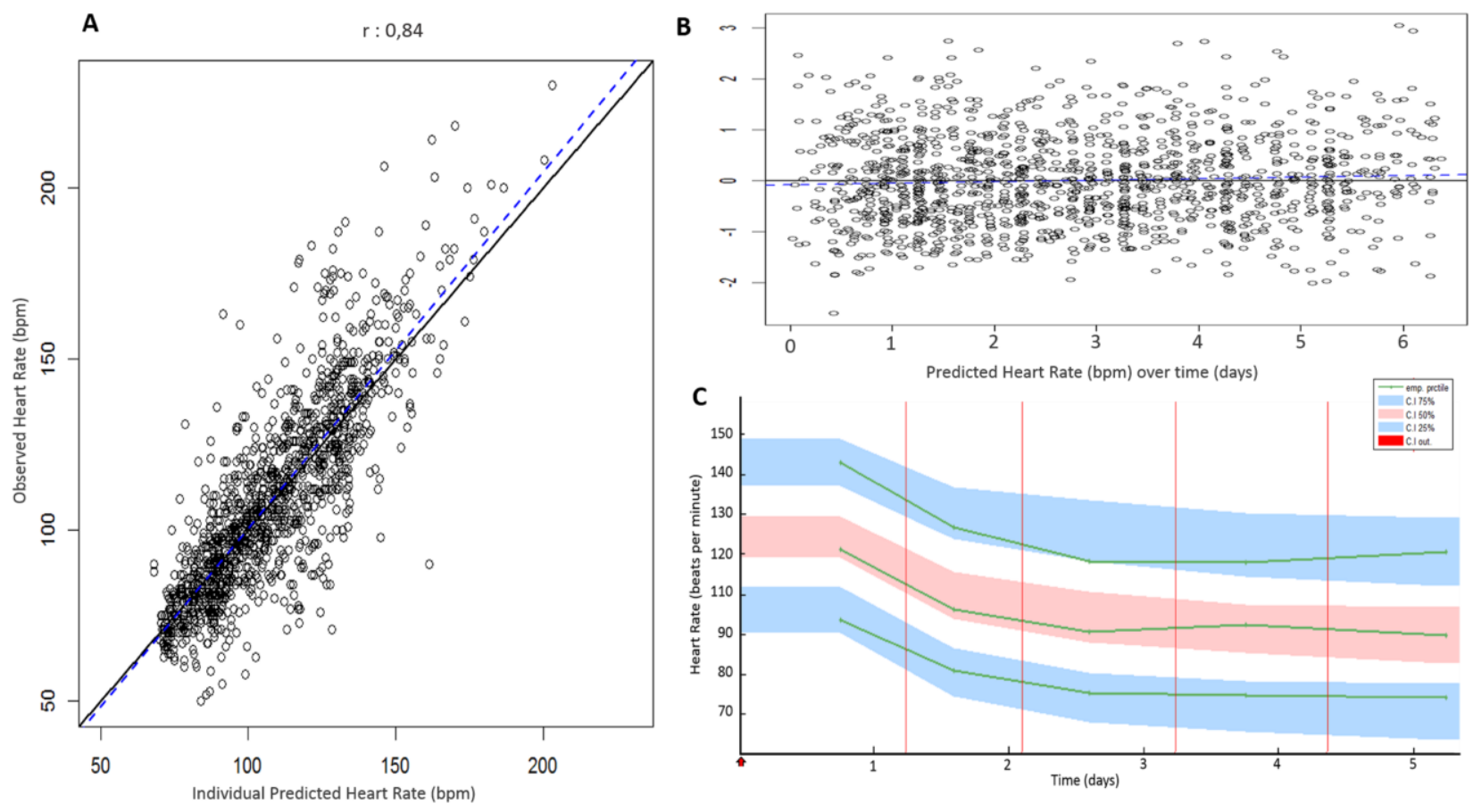


\section{FIGURE 3}

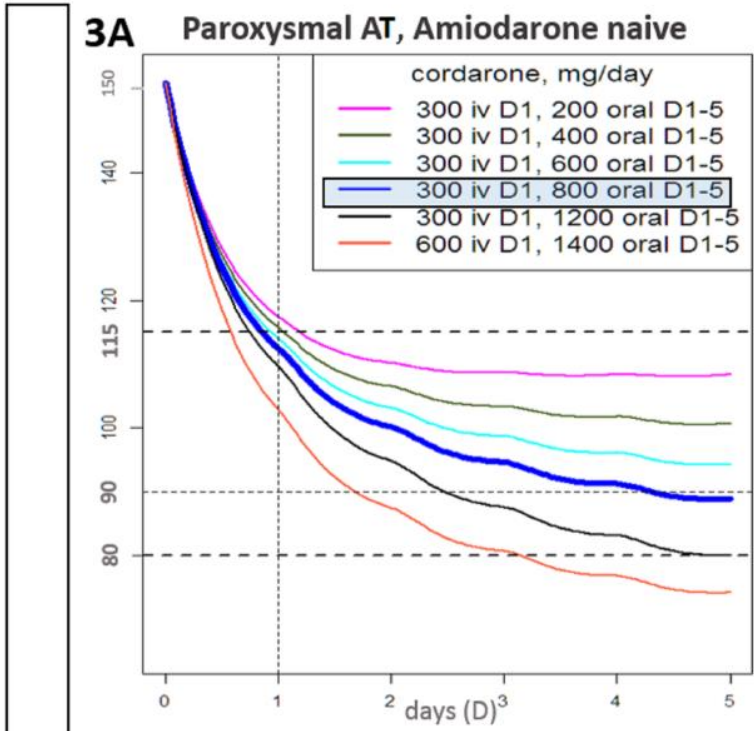

3B Paroxysmal AT, Amio Pre-treatment

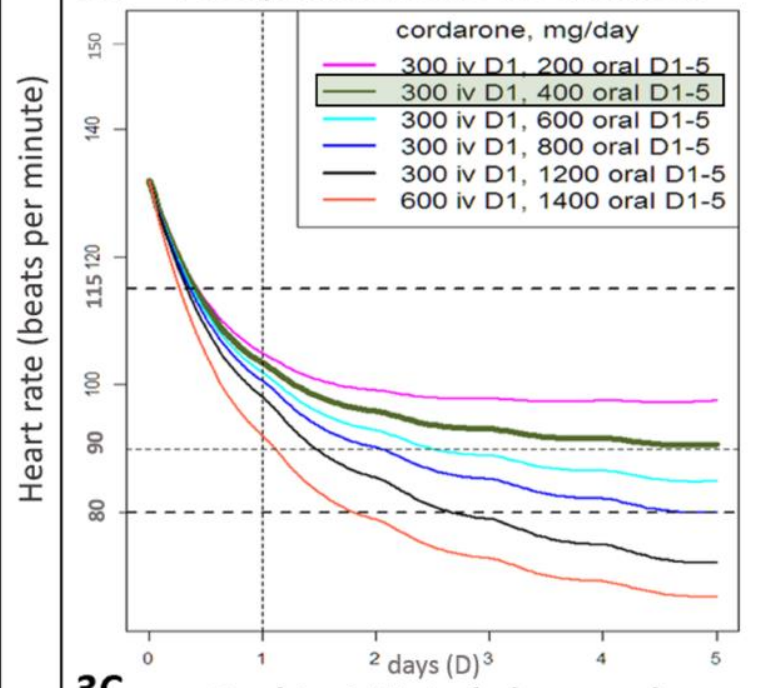

3C Persistent AT, Amiodarone naive

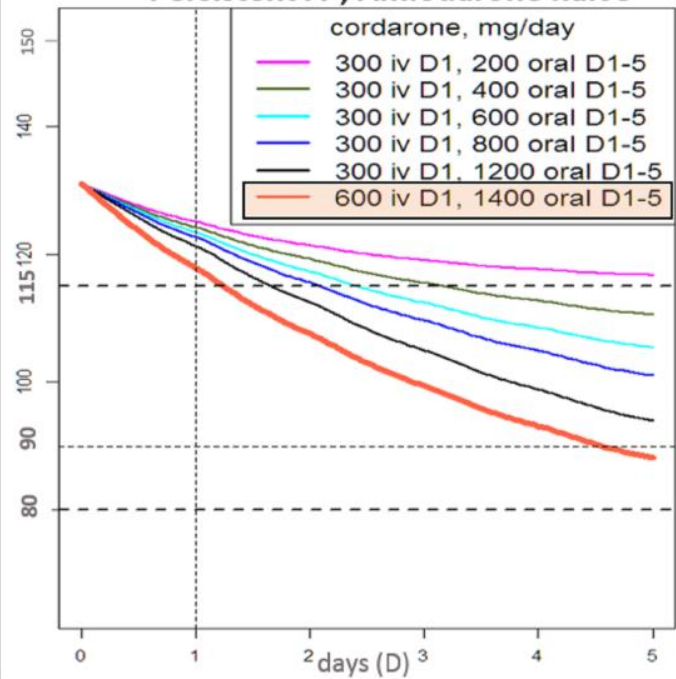

3D Paroxysmal AT, Amiodarone naive

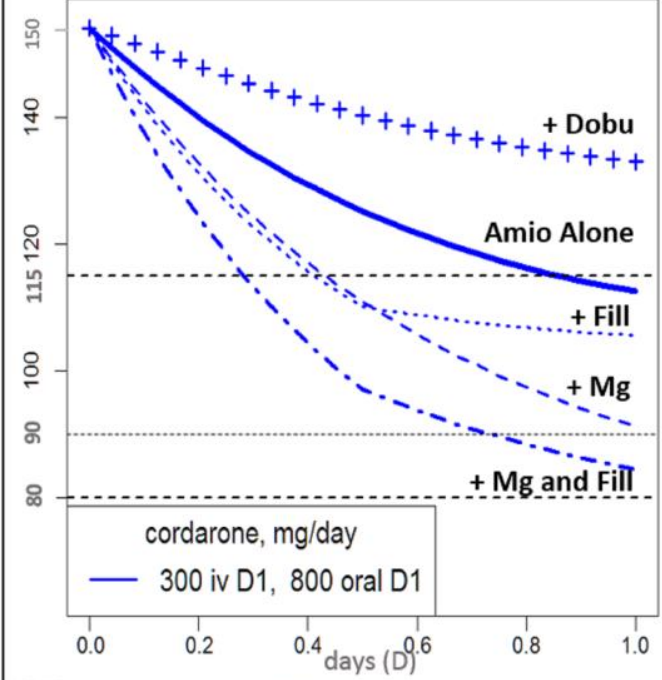

3E Paroxysmal AT, Amio Pre-treatment

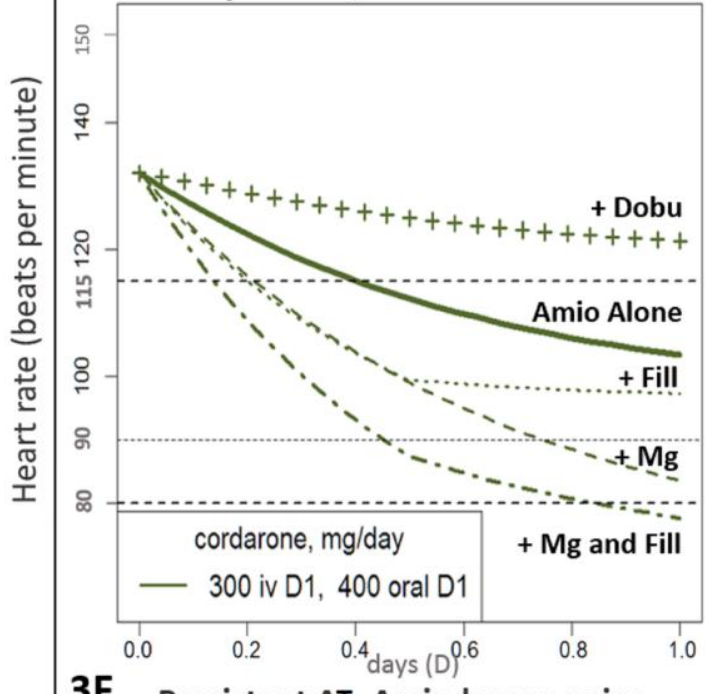

3F Persistent AT, Amiodarone naive

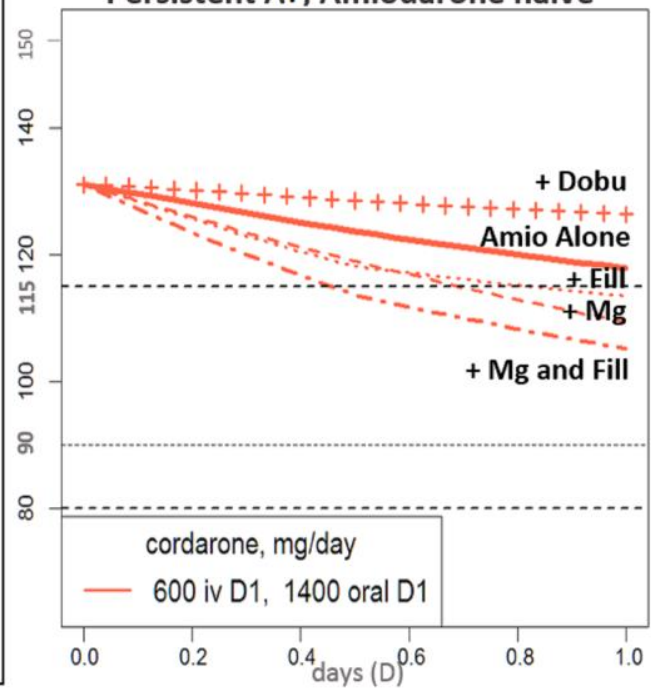

